\title{
IMAGE MOSAICING OF LOW QUALITY NEONATAL RETINAL IMAGES
}

\author{
Akhilesh Bontala, Jayanthi Sivaswamy \\ CVIT, IIIT Hyderbad, Hyderabad, India
}

Rajeev R Pappuru

\author{
LV Prasad Eye Institute, Hyderabad, India
}

\begin{abstract}
Retinopathy of prematurity (ROP) is a vascular disease in premature infants. This is characterized by abnormal vessel growth and subsequent fibrosis in the peripheral retina. The prognosis of ROP relies on information on the presence of abnormal growth and their location. Diagnosis is based on a series of images obtained with a wide field of view camera (such as RetCam), to capture the complete retina. In this paper, we present a novel and efficient hierarchal mosaicing algorithm, for neonatal images of varying quality. We employ a vessel-based quality metric and exploit the knowledge of the retinal structure to automatically select a subset of images from a given set, to construct a good quality mosaic. Such mosaics can aid the assessment of ROP by providing a comprehensive and complete view of the entire retina. The hierarchal approach underlying the method makes it possible to complete the mosaicing task in close to real time. The proposed method has been tested on 14 sets of data with each set consisting of 6-35 retinal images acquired using RetCam and the generated mosaics are found to be of good quality as validated by a clinical expert.
\end{abstract}

Index Terms - Mosaicing, Retina, ROP, Image blending

\section{INTRODUCTION}

Vascular development is complete in a normal mature baby, whereas the retinal blood vessel development continues even after birth in case of premature babies. Due to unfavorable conditions outside the mother's womb, this development can get deranged leading to abnormal retinal vascular development. This in turn triggers bleeding inside the eye, scar tissue growth and retinal detachment in the eye known as retinopathy of prematurity (ROP). As this disease is usually seen in both eyes, this is a potentially blinding condition if it is not treated in the early stage [1]. ROP is assessed using retinal images acquired using a special wide field of view camera (RetCam is an example). The complete retinal surface is captured using multiple images (views). In premature babies, imaging is difficult and the quality of acquired images is often poor due to pathological and other acquisition-related reasons. A number of parameters are used in ROP assessment [2] to estimate the prognosis and plan the treatment. The key information of interest are the vessel structure and the extent of vessel growth.

Staging of ROP requires scrutiny of the entire posterior zone in addition to the vessel tortuosity and dilation information. Current clinical practice involves mostly a manual procedure since most of the work in automated image analysis has focused only on quantifying the vessels in certain zones around the optic disk, using neonatal retinal images. Many methods (see [3] for a good review) have been proposed for interactively measuring the tortuosity and dilation of the posterior vessels. ROPtool and RISA [3] are examples of interactive tools developed. They are able to achieve high sensitivity and specificity in quantifying vessels using retinal images captured with RetCam. Both these methods rely on a good quality retinal image which is selected manually, from a set of captured images. The main limitations of the current ROP assessment procedures are all vessels in the posterior zone may or may not be visible in a single image. In such cases, even though all the required information is present in the set, these tools cannot be used as they require a single image as input. The acquired multiple views can be of high to poor quality at each location and the selected image may not contain the complete information about all the regions. Hence, others views are manually selected for obtaining the missing information. This is a laborious process and highly subjective in nature. Therefore, creation of a mosaic is of interest.

The aim of this work is to automatically create a good quality mosaic of the complete neonatal retina from a set of images acquired by RetCam type of wide-field camera. The mosaic has to capture the best available information for all the regions that have been imaged. Such a mosaic can facilitate both ROP diagnosis and its staging. We also aim to develop a technique that is fast enough so that it can provide visual feedback to technicians and prompt them to recapture regions which are not visible.

\section{BACKGROUND}

The key steps in any mosaicing algorithm are image registration, global alignment and blending of multiple images. Medical image registration has been an active research problem. In the context of adult retinal images, feature based methods are popular for image matching. Features include a Speed Up Robust Features (SURF) [4] and a variant of SIFT called msift [5]. By incorporating the color information in SIFT, robustness to color variations was achieved. A dual-bootstrap iterative closest point algorithm for matching was proposed in [6] which starts with a low-order estimate of the transformation which is accurate for a small region and an iterative refinement is done by expanding the region. A graph matching based approach has also been attempted [7] where a vessel structure graph is constructed using bifurcation points. For aligning multiple images, two main categories of algorithms have been proposed: global and sequential methods. In the former, the overall registration error between all the images is globally optimized and all the transformation parameters are simultaneously estimated [8]. In the latter category, the approach is to register an image to another image or a mosaic. In image-to-image alignment, typically, an anchor image is selected and the remaining images are registered directly or indirectly (using intermediate images) to the anchor. The overall registration error is optimized using graph algorithms with images as nodes and the registration error as the edge weights [4][9]. In the case of image-tomosaic registration, all images are registered to the mosaic one by one and the mosaic is updated after each step.

Mosaic construction from RetCam images pose several inherent challenges. Firstly, the acquired images of neonatal retina are of poor spatial resolution, variable quality and contrast as opposed to images acquired from adults. Secondly, given the difficulty in imaging a neonatal retina, different degrees of blurring (due to motion 
for example) are introduced in each image. Additionally, the overlap between the images is variable. Consequently, finding accurate matches using the popular descriptors is difficult. Thirdly, finding a global optimum or constructing a graph as in existing approaches is computationally intensive for the current problem as the number of acquired images can be large. To the best of our knowledge, no approach has been previously proposed for registration or mosaicing of neonatal retinal images.

In this paper, we address the above challenges and propose a hierarchal, hybrid approach for mosaic construction. This method is novel in three aspects. The matching of low quality and low overlapping set of images is posed as a dense correspondence problem and achieved using Radon transform based descriptor [10] proposed earlier for high resolution adult retinal images. Secondly, we gain significant reduction in computation by utilizing region based partitioning using the domain knowledge to enable handling of a large set of images. Thirdly, a final blending stage is included to fuse and improve the vessel definition information.

\section{METHOD}

Given a set of retinal images captured from different view points, our aim is to combine the information of the regions present in multiple views and create a good quality mosaic. A hierarchal approach is proposed for spatial alignment to achieve fast mosaicing. The computational steps are: 1) Partitioning - The given input image set of size $N$ is partitioned into $L$ disjoint sets (according to the region captured); 2) intermediate mosaic generation - an anchor image is chosen in each subset which is of best quality. All the images in that subset are registered to its anchor image and fused to create a set of intermediate mosaics. Fusion employs a blending mechanism which selects information based on a local quality metric 3) Final mosaic generation - Step (2) is repeated with the intermediate mosaics as input. The algorithm is presented in detail next. Fig.1 shows a schematic diagram of the proposed approach with a sample set of images.

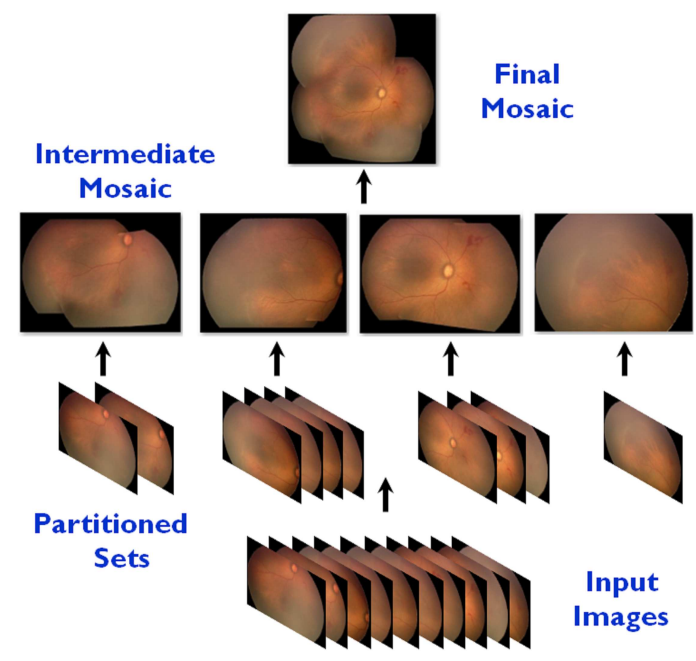

Fig. 1: Proposed hierarchal mosaicing approach

\subsection{Partitioning}

Given a set of colour images it is partitioned into disjoint sets. Formally, given $S=\left\{I_{n} ; n=1,2 . . N\right\}$ is partitioned into $S_{l} ; l=$ $1,2 . ., L \leq N ; S_{l}$ corresponds to a distinct region of the retina. $I_{n}$ is taken to be a vector valued function in all our computations. The partitioning method is based on the location information about the optic disk and the vessels. The aim of such grouping is to have maximum amount of overlapping information among images in a set, for accurate transformation estimation. A simple optic disk (OD) detection algorithm was designed to aid partitioning. This algorithm captures the color discontinuity at the boundary and accurately detects the OD even in the case of blurred and low contrast images.

An annulus-shaped template is used for OD detection. Let $R_{i}$ and $R_{o}$ be the inner and outer regions of the annulus. The radii of these regions were chosen empirically to be such that the boundary of OD lies in the annulus. At every point $p$ in the image, the template is centred at $p$ and the local maximum is determined within $R_{i}$. This is computed in the RGB space and denoted as $C_{p}$. Next, the pixel value variation relative to $C_{p}$, within the template is computed as follows:

$$
H(p)=\sum_{r \in R_{o}}\left\|I(r)-C_{p}\right\|-\sum_{r \in R_{i}}\left\|I(r)-C_{p}\right\|
$$

where $r$ is the position vector and $\|x\|$ is the $L 2$ norm of $x$. This function will be a maximum when $p$ is at the OD center. The OD detection was performed on a sub-sampled image to speed up computations. Once the OD center is found, its position is used to derive 5 partitions as follows: $S_{1}$ is the set of OD-centric images while $S_{2}$ to $S_{5}$ are the sets where OD is at the left, right, top and bottom, respectively, of the image. Images in $S$ which do not contain OD can belong only to $S_{n} ; n \neq 1$. The correct set membership is determined based on the exact location of blood vessel(s) in the peripheral region of the image and the domain knowledge. The retinal vessel network have a unique pattern as they radiate in a parabolic fashion from the $\mathrm{OD}$ in two main (nasal and temporal) directions. The next step is intermediate mosaic generation. This task requires selecting good quality images. Hence, we describe the quality assessment strategy.

\subsection{Image quality assessment}

The definition for quality of an image is task dependent. Since the primary structure of interest in ROP is the vessel network, assessing image quality based on definition of vessels within, was considered appropriate. Specific features of interest are the sharpness and contrast of the vessels which is assessed using a multi-scale ridge strength measure based on the Hessian [11] computed on the green channel. The ridge strength calculated at any point signifies the sharpness of the boundary of the vessel. In blurred regions, the transition at the boundary is smooth and hence the ridge strength is low. A vesselness measure $V$ is defined as

$$
V(p)=\left(L_{u u}^{2}-L_{v v}^{2}\right)^{2}
$$

where $u$ and $v$ are the principal directions at $p$ and $L_{u u}, L_{v v}$ are the principal curvatures. Sample patches and their $V$ maps are shown in Fig.2.

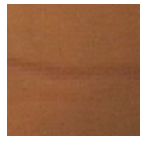

(a) Patch 1

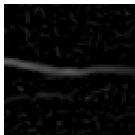

(b) $V_{1}$

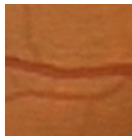

(c) Patch 2

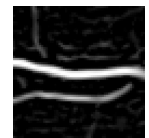

(d) $V_{2}$
Fig. 2: Sample patches (in colour) and their vesselness maps (in greyscale)

In our problem, two scenarios arise where quality assessment is needed: a global level comparison of two loosely aligned images and a local level comparison of corresponding patches of two accurately registered images. In the global comparison scenario, the images are loosely aligned and the overlapping regions nearly represent the 
same region. Hence, the sum of the values in $V(p)$ in each of the overlapping regions can be used to assess the quality. A high value indicates good quality. A similar approach can be used for comparing patches in two registered images. As these image patches represent exactly the same region, a more accurate quality comparison can be achieved. In Fig. 2, corresponding patches from two registered images and their $V$ maps are shown. It can be clearly observed that the sum of pixels values in $V$ would be high for the patch with sharp and high contrast vessels.

\subsection{Pair-wise Registration}

A preliminary task in mosaic construction is to accurately estimate the transformation between the given pair of images. The strategy we use is adapted from the approach we proposed for matching multi modal retinal images in [10]. Here, vessel bifurcation points are taken as interest points and located using the entropy of the curvature orientation histogram of a patch around a point. These interest points were inadequate to establish correspondences in RetCam images due to variable quality of the image pairs. A modified approach was designed for finding the interest points $P_{j}$. A determinant based Hessian (DOH) is computed at various scales and the scale-space maximum is found using automatic scale selection. $P_{j}$ are found by thresholding the $\mathrm{DOH}$. A low threshold is chosen to obtain a large number of interest points. These are primarily near the vasculature. A Radon based descriptor [10] is computed for each $P_{j}$ by computing the Radon transform of a patch around $P_{j}$ and row ordering the result. We choose to do this on the corresponding vessel map rather than the given image to minimize the effect of illumination changes. A bilateral matching scheme is used to find the initial correspondence. Solving a quadratic transformation model directly, with the original correspondence leads to many errors. Hence, a coarsefine approach is applied to find the accurate transformation. First, MLESAC is used to refine the correspondence by rejecting the outliers with an affine transformation assumption. Next, the transformation estimation is refined with a quadratic assumption on bi-lateral matched results which satisfy the affine transformation.

\subsection{Image Mosaicing}

The procedure for mosaicing is described in Algorithm 1. A key step in this algorithm is the fusion step required as part of step 4 . Consider a pair of registered images $F_{1}$ and $F_{2}$ with overlapping regions $F_{o 1}$ and $F_{o 2}$, respectively. The task at hand is to fuse them. The multi-band blending technique in [12] is used. A 6-level Laplacian pyramid is constructed for each $F_{i}$. A mask $M$ is derived for blending the overlapping regions $F_{o}$. The blending mask provides the key for choosing the laplacian value for $F_{o}$ depending on the quality of an image (measured as described in 3.2) relative to the other. Thus, if quality of $F_{o 1}(p)>F_{o 2}(p)$ then the laplacian of $F_{o 1}(p)$ is chosen and vice versa. The Laplacian pyramid for the blended region is constructed by applying the mask at every level. The non-overlapping regions are retained in the final mosaic. Reconstructing the image with this laplacian, results in a good quality image with continuous and smooth edges.

After combining all the images in every subset $S_{l}$ we have five intermediate mosaics. Now the problem of registering arbitrary number of images of various views is converted into registering these intermediate mosaics which have more spatial information and also possibly are of better quality than a single image. These intermediate mosaics are combined in a similar fashion. Images with the optic disk at the center (in set $S_{1}$ ) tend to be of good quality and have high overlap with the other the mosaics. Hence, the intermediate mosaic created from $S_{1}$ is chosen as the anchor image for the final stage. The rest of the mosaics are registered to the anchor

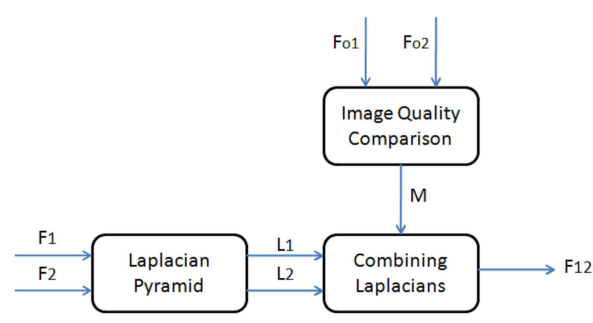

Fig. 3: Image blending

image using the same pair-wise registration algorithm. The blending mechanism is applied to combine all the registered mosaics and create the final mosaic.

Algorithm. 1: Image mosaicing algorithm
1. Partition the given set of images into disjoint sets based on
OD location
2. Select the best quality image in each subset as an anchor
image using the vesselness measure
3. Register the remaining images in the subset to the anchor
image using pair wise registration
4. Combine (with blending) the registered images in a set to
create a intermediate mosaic using local quality measure
5. Register and combine the intermediate mosaics by repeating
3 and 4 to create the complete mosaic.

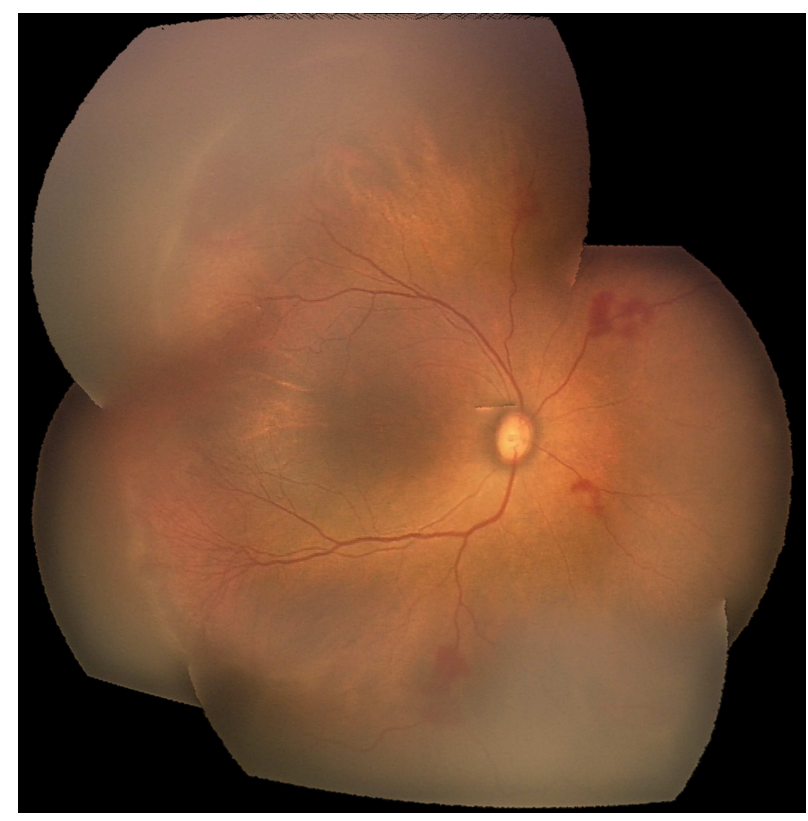

Fig. 4: A mosaic image formed using 7 retinal views from a set of 9 images

\section{EXPERIMENTS AND RESULTS}

The proposed approach was tested on 14 sets of neonatal retinal images (of size $640 \times 480$ each) obtained from various local hospitals. For each eye, 6-35 views were acquired with RetCam camera. All the cases had been diagnosed by experts as belonging to different stages of ROP. Fig. 4 shows the result of our algorithm applied to a set 9 images of which 7 images were selected by the algorithm for the final mosaic. The result of blending two sample registered 
images is shown Fig. 5. We can observe that the blended result has vessel structures which are a) present in both the input images and b) present only in one image. Specifically, the vessels present only on the right side of Image 1 and left side of the Image 2 are captured in the blended result. The overall quality is also maintained. A key feature of the proposed algorithm is that it is very fast compared to traditional graph based approaches since very few pair-wise registrations are needed. This is demonstrated by comparing the time required for computations in the two approaches. These are listed in Table 1. Here, $R$ is the unit time taken for registering two images and $M$ is the time taken for registering two intermediate mosaics. For our algorithm, $R$ was $35-50 \mathrm{sec}$ and $M$ was $75-90 \mathrm{sec}$ on a dual core 2.2 GHz Processor with 2GB RAM. A graph based method needs to compute the registration for all possible pairs and hence requires $O\left(n^{2}\right)$ registrations for combining $n$ images where as in our algorithm, the required registrations are in $O(n)$ and the upper bound on the number of mosaic-mosaic registrations is determined by the number of partitions $L$ which is 5 for our algorithm.

Table. 1: No.of pair-wise registrations needed for mosaicing

\begin{tabular}{|l|l|l|}
\hline No.of Images $(N)$ & Graph based [9] & Our method \\
\hline 6 & $9 R$ & $2 R+3 M$ \\
\hline 10 & $45 R$ & $5 R+4 M$ \\
\hline 15 & $105 R$ & $10 R+4 M$ \\
\hline
\end{tabular}

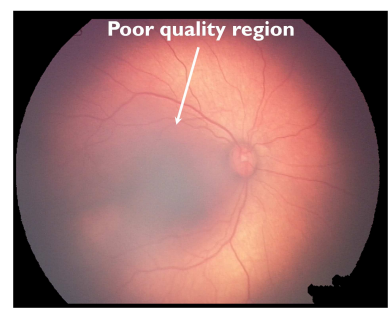

(a) Image 1

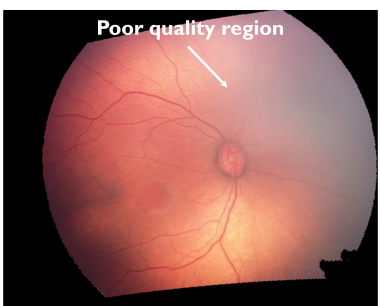

(b) Image 2

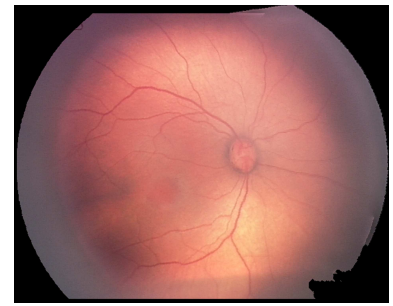

(c) After blending $1 \& 2$

Fig. 5: Result of image blending on an image pair

\section{CONCLUSION}

In this paper, we have proposed a method for neonatal retina for automatically creating a good quality mosaic of the complete retina. The mosaiced image shows a much wider field of view of the retina with better vessel structures and helps the experts in classifying ROP. The field of view in the final mosaic depends on the availability of adequate quality images. For instance, the mosaic (validated as correct by an expert) shown in Fig. 6 was constructed from a set of 31 poor quality images, of which only 15 views were selected and combined by the algorithm. It is clear that some part (top) of the retina is missing. Such visual feedback can prompt the operator to redress the missing region by reacquiring their images.

In the future, our focus will be on improving the fusion mechanism to combine the information more effectively from even poor quality images.

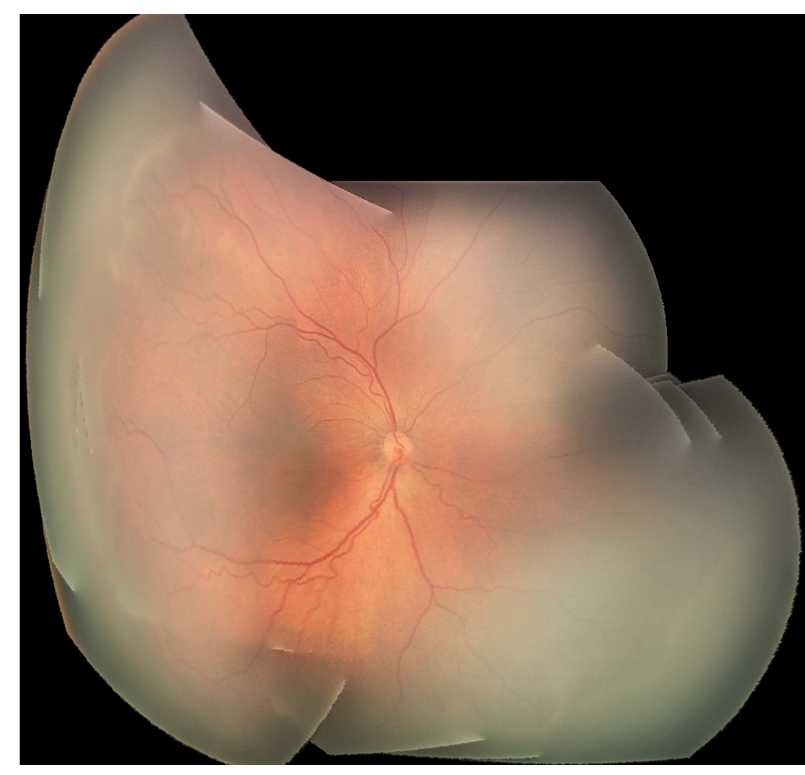

Fig. 6: Mosaic formed using 15 views from a set of 31 images

\section{REFERENCES}

[1] C Gilbert, "Retinopathy of prematurity: a global perspective of the epidemics, population of babies at risk and implications for control," Early Hum Dev, pp. 77-82, 2008.

[2] G.A. Gole et al., "The international classification of retinopathy of prematurity revisited," Archives of Ophthalmology, vol. 123, no. 7, pp. 991-999, 2005.

[3] T. Aslam et al., "Digital image analysis of plus disease in retinopathy of prematurity," Acta Ophthalmol, pp. 368-377, 2009.

[4] P. Cattin et al., "Retina mosaicing using local features," $M I C$ CAI, pp. 185-192, 2006.

[5] L. Jupeng et al., "A robust feature-based method for mosaic of the curved human color retinal images," BMEI, pp. 845-849, 2008.

[6] C.V. Stewart, C.L. Tsai, and B. Roysam, "The dual bootstrap iterative closest point algorithm with application to retinal image registration," IEEE Trans. Med. Imaging, vol. 22, no. 11, pp. 1379-1394, 2003.

[7] K. Deng et al., "Retinal fundus image registration via vascular structure graph matching," Journal of Biomedical Imaging, vol. 2010, pp. 14, 2010.

[8] A. Eden, M. Uyttendaele, and R. Szeliski, "Seamless image stitching of scenes with large motions and exposure differences," CVPR, vol. 2, pp. 2498-2505, 2006.

[9] T.E. Choe et al., "Optimal global mosaic generation from retinal images," ICPR, vol. 3, pp. 681-684, 2006.

[10] Y.B. Bathina, MV Medathati, and J. Sivaswamy, "Robust matching of multi-modal retinal images using radon transform based local descriptor," ACM IHI, pp. 765-770, 2010.

[11] T. Lindeberg, "Scale-space theory in computer vision," 1994.

[12] P.J. Burt and E.H. Adelson, "A multiresolution spline with application to image mosaics," ACM Transactions on Graphics (TOG), vol. 2, no. 4, pp. 217-236, 1983. 